Proceedings of the 43rd "Jaszowiec", International School and Conference on the Physics of Semiconductors, Wisła 2014

\title{
Polarization-Induced Band Inversion in In-Rich InGaN/GaN Quantum Wells
}

\author{
S.P. EEPKOWSKI ${ }^{a}$, W. BARDYSZEWSKI ${ }^{b}$ AND D. RODAK ${ }^{b}$ \\ ${ }^{a}$ Institute of High Pressure Physics - Unipress, Polish Academy of Sciences, \\ Sokołowska 29, 01-142 Warszawa, Poland \\ ${ }^{b}$ Faculty of Physics, University of Warsaw, L. Pasteura 5, 02-093 Warszawa, Poland
}

\begin{abstract}
We theoretically study the polarization-induced band inversion phenomenon in $c$-plane In-rich InGaN/GaN quantum wells. Our calculations performed using the $k \cdot p$ method with the $8 \times 8$ Rashba-Sheka-Pikus Hamiltonian for the structures with the indium content between $90 \%$ and $100 \%$ show that the reordering of the conduction and valence bands occurs for the quantum well widths below the theoretical values of critical thickness for InGaN layers pseudomorphically grown on GaN substrates.
\end{abstract}

DOI: 10.12693 /APhysPolA.126.1154

PACS: 73.21.Fg, 73.22.Dj

\section{Introduction}

InN and In-rich InGaN alloys have been attracting attention as important nitride semiconductors which allow to move the spectrum of light emission from the blue-UV region towards the green, red, and infrared wavelengths. This possibility is associated with the narrow band gap of InN equal approximately to $0.7 \mathrm{eV}$. Growing high-quality InGaN layers with high In content on GaN substrates is difficult due to the large lattice mismatch between $\mathrm{InN}$ and GaN. To overcome this problem ultrathin $\mathrm{InN} / \mathrm{GaN}$ quantum wells (QWs) and superlattices have been considered [1]. It has been shown theoretically by means of the $a b$ initio calculations that in these structures grown along $c$ crystalline axis, the energy gap at the $\Gamma$ point of the Brillouin zone changes dramatically with the $\mathrm{QW}$ width from about $2 \mathrm{eV}$ for 1 monolayer (ML) of $\mathrm{InN}$ to $0 \mathrm{eV}$, with inverted order of the conduction and valence band states, for 3-4 MLs of InN [2]. This effect is associated with the presence of the built-in electric fields originating from the spontaneous and piezoelectric polarizations. The magnitude of the built-in electric field in $\mathrm{InN} / \mathrm{GaN}$ QWs can reach $12-13 \mathrm{MV} / \mathrm{cm}$ which leads to a substantial potential drop over the $\mathrm{InN}$ region even for a few MLs thin QWs. The band inversion predicted for $\mathrm{InN} / \mathrm{GaN}$ QWs with 4 MLs of InN was used to suggest the possibility of existence of a topological insulator state in these structures [2].

In this work, we extend the theoretical study of the polarization-induced band inversion phenomenon to c-plane In-rich InGaN/GaN QWs. Our calculations performed for the structures with the indium content between $90 \%$ and $100 \%$ show that the reordering of the conduction and valence bands occurs for the QW thicknesses below the theoretical values of critical thickness for pseudomorphic growth of $\operatorname{In}_{x} \mathrm{Ga}_{1-x} \mathrm{~N} / \mathrm{GaN}$ QWs.

\section{Theoretical model}

In order to describe the electronic band structure of indium rich $\mathrm{InGaN} / \mathrm{GaN}$ QWs we use the $k \cdot p$ method with the $8 \times 8$ Rashba-Sheka-Pikus Hamiltonian, which takes into account the coupling between the conduction states of $\Gamma_{7}$ symmetry and valence band states transforming according to the $\Gamma_{9}$ (heavy holes) and $\Gamma_{7}$ (light and spin-orbit split-off holes) symmetries. The band structure parameters for this Hamiltonian have been obtained by interpolating the literature data for $\mathrm{GaN}$ and $\mathrm{InN}$ from Refs. [2] and [3]. In order to take into account the broken translational symmetry in the direction perpendicular to the QW plane, the $z$-component of momentum in the Hamiltonian is replaced by the $-\mathrm{i} \hbar \frac{\partial}{\partial z}$ operator. Resulting system of 8 differential equations is solved using the finite element method. Strain and the built-in electric field entering the Hamiltonian are determined using the nonlinear theory of elasticity and piezoelectricity including contributions arising from the second-order piezoelectric constants and the third-order elastic constants [4].

\section{Results}

The band structure calculations have been performed for InGaN/GaN QWs lattice matched to GaN substrate with the In content equal to $100 \%, 95 \%$, and $90 \%$. The QW width has been changed from $1.3 \mathrm{~nm}$ to $2 \mathrm{~nm}$, while the barrier thickness was kept at $40 \mathrm{~nm}$.

In Fig. 1a and b, we show the electronic band structures in the vicinity of the $\Gamma$ point for two $\mathrm{InN} / \mathrm{GaN}$ QW structures with the QW width equal to 1.4 and $1.6 \mathrm{~nm}$, respectively. Situation presented in Fig. 1a corresponds to the normal ordering of bands with the $\Gamma_{7}$ conduction band states (grey line) lying above the valence bands states (black lines), whereas in Fig. 1b the ordering of bands is reversed for small values of the in-plane wave vector $\boldsymbol{k}_{\|}$. It is worth noting that for large values of $\boldsymbol{k}_{\|}$, the ordering of bands is restored to the normal band structure.

In Fig. 2 we present the dependence of the band energy difference at the $\Gamma$ point between the $\Gamma_{7}$ conduction band state and the $\Gamma_{9}$ valence band state on the $\mathrm{QW}$ width for $\mathrm{InN} / \mathrm{GaN}, \mathrm{In}_{0.95} \mathrm{Ga}_{0.05} \mathrm{~N} / \mathrm{GaN}$ and $\operatorname{In}_{0.9} \mathrm{Ga}_{0.1} \mathrm{~N} / \mathrm{GaN}$ 


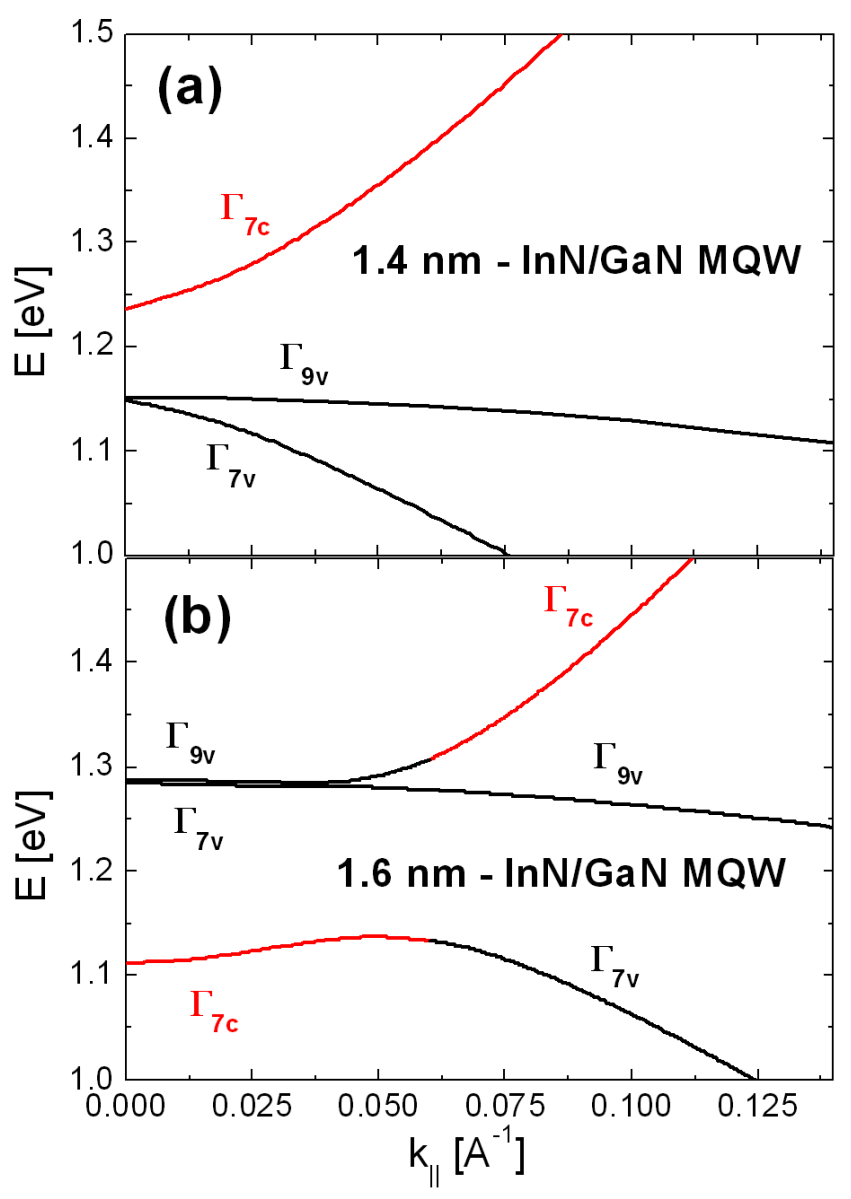

Fig. 1. Band structure of InN/GaN QWs in the vicinity of the band gap for the QW width of (a) $1.4 \mathrm{~nm}$ and (b) $1.6 \mathrm{~nm}$. The grey (red on-line) color reflects the states with the dominant contribution from the conduction band $s$-type states, while the black color corresponds to the dominant valence band $p$-states.

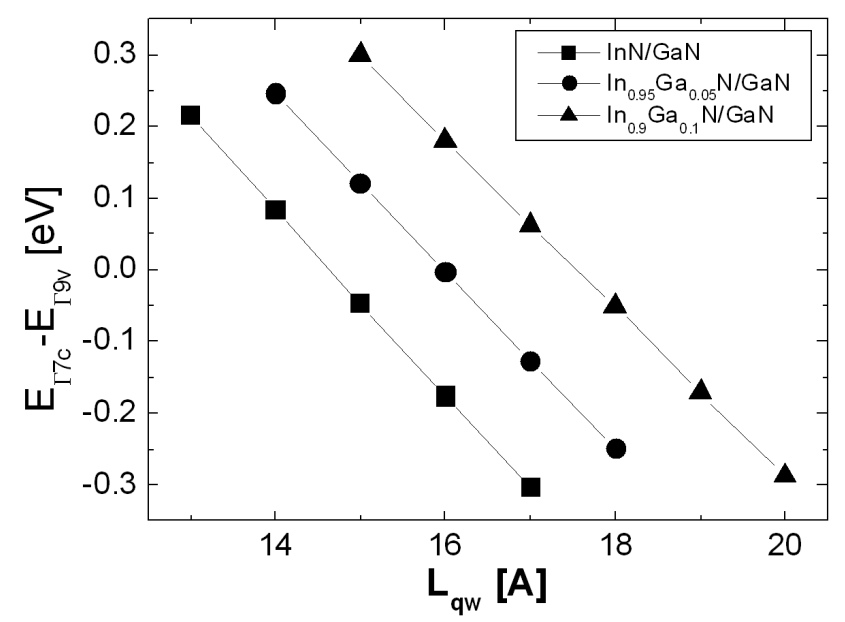

Fig. 2. The dependence of the difference between the $\Gamma_{7}$ conduction band state and the $\Gamma_{9}$ valence band state on the QW width in $\mathrm{InN} / \mathrm{GaN}, \mathrm{In}_{0.95} \mathrm{Ga}_{0.05} \mathrm{~N} / \mathrm{GaN}$, and $\mathrm{In}_{0.9} \mathrm{Ga}_{0.1} \mathrm{~N} / \mathrm{GaN}$ QW structures.
QW structures. It can be observed that for InN/GaN QWs, the transition from the normal to inverted band structure occurs at the QW thickness equal to $1.5 \mathrm{~nm}$ which corresponds very well to the results presented in Ref. [2]. Increasing Ga content in the QW region shifts the critical QW width corresponding to the band inversion to $1.6 \mathrm{~nm}$ and $1.8 \mathrm{~nm}$ for $\mathrm{In}_{0.95} \mathrm{Ga}_{0.05} \mathrm{~N} / \mathrm{GaN}$ and $\mathrm{In}_{0.9} \mathrm{Ga}_{0.1} \mathrm{~N} / \mathrm{GaN}$, respectively. The dependence of the energy gap on the QW width has almost the same slope in all three cases.

\section{Conclusions}

We have analyzed the polarization-induced band inversion effect in In-rich, fully strained $\mathrm{In}_{x} \mathrm{Ga}_{1-x} \mathrm{~N} / \mathrm{GaN}$ QWs. Our calculations performed for the structures with the indium content between $90 \%$ and $100 \%$ have shown that the reordering of bands occurs for the QW widths below the theoretical values of critical thickness for pseudomorphic growth of $\operatorname{In}_{x} \mathrm{Ga}_{1-x} \mathrm{~N} / \mathrm{GaN}$ QWs [2]. Therefore, obtaining fully strained indium rich $\mathrm{In}_{x} \mathrm{Ga}_{1-x} \mathrm{~N} / \mathrm{GaN}$ QWs with inverted band structures seems to be experimentally available.

\section{Acknowledgments}

S.モ. thanks K. Sakowski for useful discussion concerning the finite element method. This work was supported by the Polish National Science Center, Project No. 2012/07/B/ST3/03174.

\section{References}

[1] M.S. Miao, Q.M. Yan, C.G. Van de Walle, Appl. Phys. Lett. 102, 102103 (2013).

[2] M.S. Miao, Q.M. Yan, C.G. Van de Walle, W.K. Lou, L.L. Li, K. Chang, Phys. Rev. Lett. 109, 186803 (2012).

[3] P. Rinke, M. Winkelnkemper, A. Qteish, D. Bimberg, J. Neugebauer, M. Scheffler, Phys. Rev. B 77, 075202 (2008).

[4] J. Pal, G. Tse, V. Haxha, M.A. Migliorato, S. Tomic, Phys. Rev. B 84, 085211 (2011). 